\title{
CS Research Square \\ Is immediate weight bearing safe for lateral malleolus fracture after surgery?
}

\section{Sang-June Lee}

CHA Bundang Medical Center, CHA University https://orcid.org/0000-0003-0582-7413

Young Rak Choi ( $\nabla$ jeanguy@hanmail.net )

SeongJu Choi

CHA Bundang Medical Center

Ho Seong Lee

Asan Medical Center

Seung-Hwan Park

Asan Medical Center

\section{Research article}

Keywords: Ankle fracture, Lateral malleolus fracture, Early rehabilitation, Immediate weight bearing

Posted Date: February 27th, 2020

DOI: https://doi.org/10.21203/rs.2.24701/v1

License: (c) (i) This work is licensed under a Creative Commons Attribution 4.0 International License.

Read Full License

Version of Record: A version of this preprint was published at Foot \& Ankle Orthopaedics on October 1st, 2020. See the published version at https://doi.org/10.1177/2473011420S00167. 


\section{Abstract}

Introduction Lateral malleolus fracture is one of the most common fractures. However, there is controversy regarding the rehabilitation protocols used after surgery. In particular, the initiation point for weight bearing has not been standardized. In the present study, we investigated the prognostic difference between immediate and delayed weight bearing on lateral malleolus fractures.

Materials and Methods The medical records of matched 50 and 41 patients in the immediate and delayed weight-bearing groups were reviewed retrospectively. All patients were treated with open reduction and internal fixation using an anatomical locking compression plate. In the immediate weightbearing group (IWB), tolerable weight bearing (i.e., can be endured immediately after surgery with crutches) was permitted. In the delayed weight-bearing group (DWB), weight bearing was completely restricted for 4 weeks after surgery. Ankle motion exercise was permitted in both groups starting from the day after surgery. Radiographic assessment data and clinical outcomes were reviewed.

Results No significant differences in the radiographic assessments and complications were found between the two groups. Significant differences in shortened length of hospital stay and time to return to work with the IWB rehabilitation protocol were confirmed (IWB group vs. DWB group: 5.7 vs. 8.0 days and 6.0 vs. 8.0 months, respectively). A significant difference in sport factor was observed in the Foot and Ankle Outcome Score at 3 months postoperatively (75.5 vs 68.5).

Conclusions We found no significant differences between the two groups with respect to postoperative radiological outcome and complications. The benefits of shortening the time to return to work and length of hospital stay associated with the IWB rehabilitation protocol were confirmed. In conclusion, immediate weight bearing is recommended in patients with lateral malleolus fracture after anatomical reduction and firm fixation by surgery.

\section{Introduction}

Ankle fracture is one of the most commonly reported fractures. Population-based epidemiological studies have shown an incidence of 168.7 in 100,000 fractures/year over a decade. The most common type of fracture in all age groups was lateral malleolus fracture, representing $55 \%$ of all ankle fractures [1]. During normal gait, the tibia plays the major role rather than the fibula. With the ankle joint in neutral position, the weight distribution of the fibula reached $6.4 \%$. With the anatomical characteristics in weight bearing at the fibula and the development of plates, patients with this type of structure showed improved postoperative outcome. Immediate weight bearing (IWB) after surgery has been suggested in this setting. However, the initiation point for weight bearing during postoperative rehabilitation is controversial.[2, 3]

Studies have suggested that early postoperative weight bearing should be performed to prevent the development of iatrogenic joint stiffness, muscle atrophy, and deep vein thrombosis $[4,5]$. In addition, Simanski et al. suggested no disadvantages with early weight bearing to delayed weight bearing (DWB) concerning the duration of hospitalization, pain intensity, time until return to work, and clinical scores [6]. 
According to a systematic review, no significant differences were found between the two approaches in terms of function, pain, range of motion, radiological assessment, complications, and return to work $[7,8]$.

By contrast, other studies reported that postoperative IWB should be restricted to protect against soft tissue damage and loss of reduction [9]. Many surgeons assume that early weight bearing should be applied as soon as possible after surgery, but this application is limited owing to the lack of experience or concerns of displacement.

Therefore, the aim of the present study was to determine the potential prognostic differences between IWB and DWB in patients with a lateral malleolus fracture surgically treated with anatomical reduction and firm fixation.

\section{Materials And Methods}

The medical records of 220 patients who underwent open reduction and internal fixation (ORIF) of the unilateral ankle fracture were retrospectively investigated. The procedures were performed by a single surgeon from January 2015 to April 2017. Among the 220 patients, 180 had a lateral malleolus fracture treated with ORIF using an anatomical locking compression plate (LCP). All the patients who underwent surgery showed one or more of the following unstable findings before surgery as a surgical indication: displacement of $>2 \mathrm{~mm}$, fibular shortening, rotation, unbearable weight bearing and incongruent ankle mortise. Stress radiographic view was not performed because of pain. Patients with open ankle fracture, unavailable follow-up data for $>12$ months and low compliance and combined ankle injury including definite deltoid ligament injury and osteochondral fracture of the talus were excluded from the analysis. Finally, 91 patients who had lateral malleolus fractures treated using anatomical LCP were included (Fig. 1). Matching was performed for sex, age, underlying diseases, and other combined injuries. As a result, of the patients, 50 underwent IWB after surgery (IWB group), whereas 41 did not undergo weight bearing for 4 weeks after surgery (DWB group). Both postoperative rehabilitation protocols were implemented randomly. During the period between 2015 and 2016, we applied DWB protocol. Since then, IWB protocol has been applied. No different specific criteria regarding fracture pattern and fixation material were used for applying the protocols in the patients except the timing factor. Anatomical reduction was defined as an intraoperative fracture gap after fixation of $\leq 1 \mathrm{~mm}$. Firm fixation was evaluated intraoperatively through observation of the fracture site with ankle joint motion.

\section{Postoperative management and rehabilitation}

After surgery, all the patients received a walking brace for 4 weeks. In the IWB group, tolerable weight bearing (i.e., that can be endured immediately after surgery with crutches) was permitted. At that time, pain was controlled and not excessive, but tolerable rehabilitation was recommended. One week after surgery, most patients tolerated the weight without difficulty. Four weeks after surgery, in the outpatient department, almost all the patients achieved full weight bearing. In the DWB group, weight bearing was completely restricted for 4 weeks after surgery. After this period, tolerable weight bearing was initiated. 
The patients in the DWB group who reported pain during weight bearing 4 weeks after surgery were treated with a similar protocol to that applied in the IWB group on the first week. Ankle motion exercise was permitted in both groups on the day after surgery (Fig. 2).

\section{Outcome measure}

Ankle radiographs (i.e., in the anteroposterior, lateral, and mortise planes) were evaluated on the day after surgery and at 3 and 12 months after surgery. The radiographic assessment was focused on the time of bony union, loss of reduction, and formation of a new fracture. Loss of reduction was defined as a translation of $>2 \mathrm{~mm}$ as compared with the postoperative immediate plain radiographs in 2 of 3 planes (anteroposterior, lateral, and mortise plane) at 3 and 12 months after operation. Moreover, bony union was defined as the bridging of the trabeculae or osseous bone in 2 of the 3 planes (Fig. 3).

Clinically, the duration of hospitalization, time to return to work, and complications, especially surgical site pain, infection, wound dehiscence, and deep vein thrombosis, were evaluated. Surgical site pain was defined as pain (i.e., $>5$ points on the visual analogue scale) prolonged for $>2$ weeks after surgery. As clinical score, the Foot and Ankle Outcome Score (FAOS) was evaluated at 3 and 12 months after surgery.

\section{Statistical analyses}

The demographic and clinical variables of the 2 groups were compared using the chi-square or Fisher exact test (categorical variables), and the Student t-test or analysis of variance (continuous variables). The Mann-Whitney U test was used to evaluate differences between the two groups. A p value of $<0.05$ denoted statistical significance. Data were analyzed using the SPSS version 21.0 statistical software package (IBM Corp., Armonk, NY, USA).

\section{Results}

A total of 91 patients were evaluated. Fifty and 40 patients underwent IWB and DWB, respectively. The mean ages of the patients in the IWB and DWB groups were 44.7 years (range: 25-66 years) and 49.2 years (range: $20-60$ years), respectively. In addition, the mean follow-up durations were 16.5 months (range: 14-22 months) and 20.4 months (range: 15-25 months), respectively. According to the DanisWeber classification, the most frequent type of fracture was B (i.e., 46 and 34 fractures, respectively). No significant differences were found between the 2 groups regarding sex, age, number of total screws, and fracture classification (Table 1). 
Table 1

Characteristics of the patients

\begin{tabular}{|llll|}
\hline & IWB & DWB & p-value \\
\hline Number of patients & 50 & 41 & \\
\hline Sex (male/female) & $20 / 30$ & $15 / 26$ & 0.74 \\
\hline Age (years) & $44.7[25-66]$ & $49.2[20-60]$ & 0.24 \\
\hline Weber-A/Weber-B/Weber-C & $4 / 46 / 0$ & $4 / 34 / 3$ & 0.14 \\
\hline Mean follow-up period (months) & $16.5[14-22]$ & $20.4[15-25]$ & 0.12 \\
\hline Number of screws & $7.2[7-10]$ & $8.1[7-9]$ & 0.18 \\
\hline IWB: immediate weight-bearing; DWB: delayed weight-bearing & \\
\hline
\end{tabular}

Significant differences in the length of hospital stay and time to return to work between the 2 groups were confirmed (IWB group vs. DWB group: 5.7 vs. 8.0 days and 6.0 vs. 8.0 months, respectively). On the plain radiographs, the time of bony union did not show significant differences. The mean times to bony union were 4.4 and 4.3 months, respectively $(p=0.12)$. Regarding complications, several patients reported surgical site pain ( 4 and 0 cases, respectively; $p=0.06$ ), surgical site infection ( 1 and 2 cases, respectively; $p=0.44$ ), and wound dehiscence ( 1 and 2 cases, respectively; $p=0.44)$. Notably, loss of reduction, formation of a new fracture, nonunion, and malunion were not reported in any of the patients. In the FAOS, a significant difference in sport factor was found at 3 months after operation. The FAOSs were 75.5 and 68.5 , respectively $(p=0.04$; Table 2$)$. 
Table 2

Outcome measures

\begin{tabular}{|c|c|c|c|}
\hline & IWB & DWB & p-value \\
\hline \multicolumn{4}{|l|}{ Radiographic assessment } \\
\hline Time to bony union (months) & $4.4[3-6.3]$ & $4.3[2.8-6.2]$ & 0.12 \\
\hline Loss of reduction (3 and 12 months) & $0 / 50(0 \%)$ & 0/41 (0\%) & 1.00 \\
\hline $\begin{array}{l}\text { Formation of a new fracture } \\
\text { ( } 3 \text { and } 12 \text { months) }\end{array}$ & $0 / 50(0 \%)$ & $0 / 41(0 \%)$ & 1.00 \\
\hline \multicolumn{4}{|l|}{ Clinical outcome } \\
\hline Hospitalization duration (days) & $5.7[5-9]$ & $8.0[6-10]$ & $0.04 *$ \\
\hline Time to return to work (months) & $6.0[4.2-12.2]$ & $8.0[7.2-8.5]$ & $0.03 *$ \\
\hline Surgical site pain & $4 / 50(8 \%)$ & $0 / 41(0 \%)$ & 0.06 \\
\hline Surgical site infection & $1 / 50(2 \%)$ & $2 / 41(5 \%)$ & 0.44 \\
\hline Wound dehiscence & $1 / 50(2 \%)$ & $2 / 41(5 \%)$ & 0.44 \\
\hline Deep vein thrombosis & $0 / 50(0 \%)$ & $0 / 41(0 \%)$ & 1.00 \\
\hline FAOS for pain (3 months) & $65.8[60.3-70.5]$ & $70.2[65.5-71.3]$ & 0.06 \\
\hline FAOS for symptom (3 months) & $70.3[68.2-73.2]$ & $71.2[63.8-78.1]$ & 0.34 \\
\hline FAOS for ADL (3 months) & $75.5[69.9-78.2]$ & $73.3[70.2-75.3]$ & 0.32 \\
\hline FAOS for sport (3 months) & $75.5[65.2-85.3]$ & $68.5[65.3-75.3]$ & $0.04 *$ \\
\hline FAOS for QOL (3 month) & $75.3[65.3-79.8]$ & $74.3[68.3-77.7]$ & 0.15 \\
\hline FAOS for pain (1 year) & $89.3[81.3-93.5]$ & $88.3[83.5-88.5]$ & 0.68 \\
\hline FAOS for symptom (1 year) & $86.3[82.2-90.3]$ & $83.5[80.3-88.2]$ & 0.16 \\
\hline FAOS for ADL (1 year) & $90.5[85.3-95.3]$ & $89.5[80.2-95.3]$ & 0.53 \\
\hline FAOS for sport (1 year) & $93.3[85.3-96.6]$ & $90.2[85.3-97.3]$ & 0.32 \\
\hline FAOS for QOL (1 year) & $90.3[85.3-95.6]$ & $88.8[82.3-90.3]$ & 0.60 \\
\hline
\end{tabular}

\section{Discussion}


After the long period of development of surgery and devices for the treatment of lateral malleolus fracture, surgical techniques have been standardized as anatomical reduction and internal firm fixation. However, the postoperative rehabilitation protocols currently used vary considerably. Thus far, only a limited number of specific rehabilitation studies have assessed this approach according to fracture site and pattern.

A cadaveric study demonstrated no significant fracture displacement, hardware failure, or occurrence of new fractures in cases of unstable ankle fracture after ORIF with an axial compression load [10]. When applying early weight bearing in patients, surgeons should exercise caution regarding the displacement of the fracture site and union. Such concerns may interfere with the decision to apply this rehabilitation approach, especially for less experienced surgeons.

The initiation of weight bearing is accelerated for numerous reasons. First, the application of early weight bearing may accelerate the return of the patient to independent daily life and work. Gul et al. reported that patients who underwent early weight bearing were associated with a significantly more rapid return to work than those who did not undergo early weight bearing. In the present study, significant differences in the shortened hospitalization duration and time to return to work were observed (IWB group vs. DWB group: 5.7 vs. 8.0 days and 6.0 vs. 8.0 months, respectively). Second, joint stiffness may be prevented. Sondenaa et al. confirmed limited ankle range of motion in a postoperative ankle fracture immobilized for 6 weeks with plaster cast. In our scoring system, we found a significant difference in the sport factor evaluated at 3 months after surgery (IWB group vs. DWB group: 75.5 vs. 68.5 points, respectively). We assumed that the patient was unable to exercise because of the limitation of joint motion. Finally, other advantages (i.e., prevention of muscle wasting and osteoporosis) are expected. However, these effects have not been investigated thus far $[11,12]$.

In our study, we found no significant differences in postoperative radiological outcome and complications between the two groups. Notably, fewer patients had complications (i.e., infection, wound problem, displacement, and delayed union) in the IWB and DWB groups, respectively. These findings demonstrate that the IWB rehabilitation protocol has more advantages than the DWB protocol. Furthermore, we confirmed the benefits of shortening the time to return to work and hospital stay with the IWB rehabilitation protocols. Our study conclusively revealed that these benefits and safety assessment may have significant impacts on the decision of surgeons to allow weight bearing.

This study was characterized by limitations. First, we could not obtain sufficient data regarding functional outcome. Data related to range of joint motion and time of full weight bearing would be helpful in confirming the benefit and verifying the hypothesis. As parameters of muscle weakness and osteoporosis, muscle mass and bone density after rehabilitation protocol must also be measured in future studies. Second, we could not assess patient compliance. Although the differences were not statistically significant, 4 patients in the IWB group reported postoperative pain after weight bearing for 2 weeks after surgery. Although this was not confirmed, the occurrence of pain may have affected the compliance of the patients. Therefore, investigators should devise strategies to improve patient 
compliance. Third, the study design is the retrospective chart review, which makes it impossible to randomly assign the patients into two groups. However, since the rehabilitation protocol was applied differently as IWB starting from 2016, there is no selection bias. Finally, our study only involved patients with lateral malleolar ankle fractures. On the basis of the data obtained from a randomized controlled trial, the postoperative rehabilitation method for each fracture pattern should be standardized.

In our study, we found no significant differences between the two groups in terms of postoperative radiological outcomes and complications. The benefits of shortening the time to return to work and hospital stay with the IWB rehabilitation protocol were confirmed. In conclusion, application of immediate weight bearing after surgery is recommended for patients with a lateral malleolus fracture who had undergone anatomical reduction and firm fixation.

\section{Abbreviations}

IWB: Immediate weight-bearing, DWB: Delayed weight-bearing, FAOS: Foot and Ankle Outcome Score

\section{Declarations}

\section{Ethics approval and consent to participation}

All the procedures performed in the studies involving human participants were in accordance with the ethical standards of the institutional research committee (IRB registration No. CHAMC201903062) and the principles of the 1964 Declaration of Helsinki and its later amendments or comparable ethical standards. The institutional review board approved this retrospective study.

\section{Consent for publication}

The approval of the Institutional Review Board included the consent for publication of any individual data.

\section{Availability of data and materials}

The datasets of the present study are available from the corresponding author on reasonable request.

\section{Competing interests}

The author declares that they have no competing interests. 


\section{Funding}

This research was partially supported by the Korea Health Technology R\&D Project through the National Research Foundation of Korea (NRF) grant funded by the Korea government (NRF-2017R1C1B5017705).

\section{Author's contributions}

SJ Lee wrote the article and reviewed the article

YR Choi performed the surgical procedures and coordinated the study.

SJ Choi did the statistical analysis.

HS Lee coordinated the study.

SH Park did the statistical analysis.

\section{Acknowledgements}

Not applicable.

\section{References}

1. Elsoe R, Ostgaard SE, Larsen P. Population-based epidemiology of 9767 ankle fractures. Foot Ankle Surg. 2018;24:34-9.

2. Petrisor BA, Poolman R, Koval K, Tornetta III P, Bhandari M, trauma E-BOTWGJJoo. Management of displaced ankle fractures. J Orthop Trauma. 2006;20:515-8.

3. Hoelsbrekken SE, Kaul-Jensen K, Mørch T, Vika H, Clementsen T, Paulsrud Ø, Petursson G, Stiris M, Strømsøe KJJoot. Nonoperative treatment of the medial malleolus in bimalleolar and trimalleolar ankle fractures: a randomized controlled trial. J Orthop Trauma. 2013;27:633-7.

4. Harager K, Hviid K, Jensen CM, Schantz K. Successful immediate weight-bearing of internal fixated ankle fractures in a general population. J Orthop Sci. 2000;5:552-4.

5. Gul A, Batra S, Mehmood S, Gillham NJAob. Immediate unprotected weight-bearing of operatively treated ankle fractures. Journal of Acta orthopaedica belgica. 2007;73:360.

6. Simanski CJ, Maegele MG, Lefering R, Lehnen DM, Kawel N, Riess P, Yucel N, Tiling T, Bouillon B. Functional treatment and early weight bearing after an ankle fracture: a prospective study. $\mathrm{J}$ Orthop 
Trauma. 2006;20:108-14.

7. Black JD, Bhavikatti M, Al-Hadithy N, Hakmi A, Kitson J. Early weight-bearing in operatively fixed ankle fractures: a systematic review. Foot (Edinb). 2013;23:78-85.

8. Smith TO, Davies L. When should open reduction and internal fixation ankle fractures begin weight bearing? A systematic review. Eur J Trauma Emerg Surg. 2008;34:69-76.

9. Lehtonen $\mathrm{H}$, Järvinen TL, Honkonen S, Nyman M, Vihtonen K, Järvinen MJJ. Use of a cast compared with a functional ankle brace after operative treatment of an ankle fracture: a prospective, randomized study. J Bone Joint Surg Am. 2003;85:205-211.

10. Tan EW, Sirisreetreerux N, Paez AG, Parks BG, Schon LC, Hasenboehler EA. Early weightbearing after operatively treated ankle fractures: A biomechanical analysis. Foot Ankle Int. 2016;37:652-8.

11. Dehghan N, McKee MD, Jenkinson RJ, Schemitsch EH, Stas V, Nauth A, Hall JA, Stephen DJ, Kreder HJJJoot. Early weightbearing and range of motion versus non-weightbearing and immobilization after open reduction and internal fixation of unstable ankle fractures: a randomized controlled trial. $J$ Orthop Trauma. 2016;30:345-352.

12. Smeeing DP, Houwert RM, Briet JP, Kelder JC, Segers MJ, Verleisdonk EJM, Leenen LP, Hietbrink FJPO. Weight-bearing and mobilization in the postoperative care of ankle fractures: a systematic review and meta-analysis of randomized controlled trials and cohort studies. Plos one 10:e0118320.

\section{Figures}




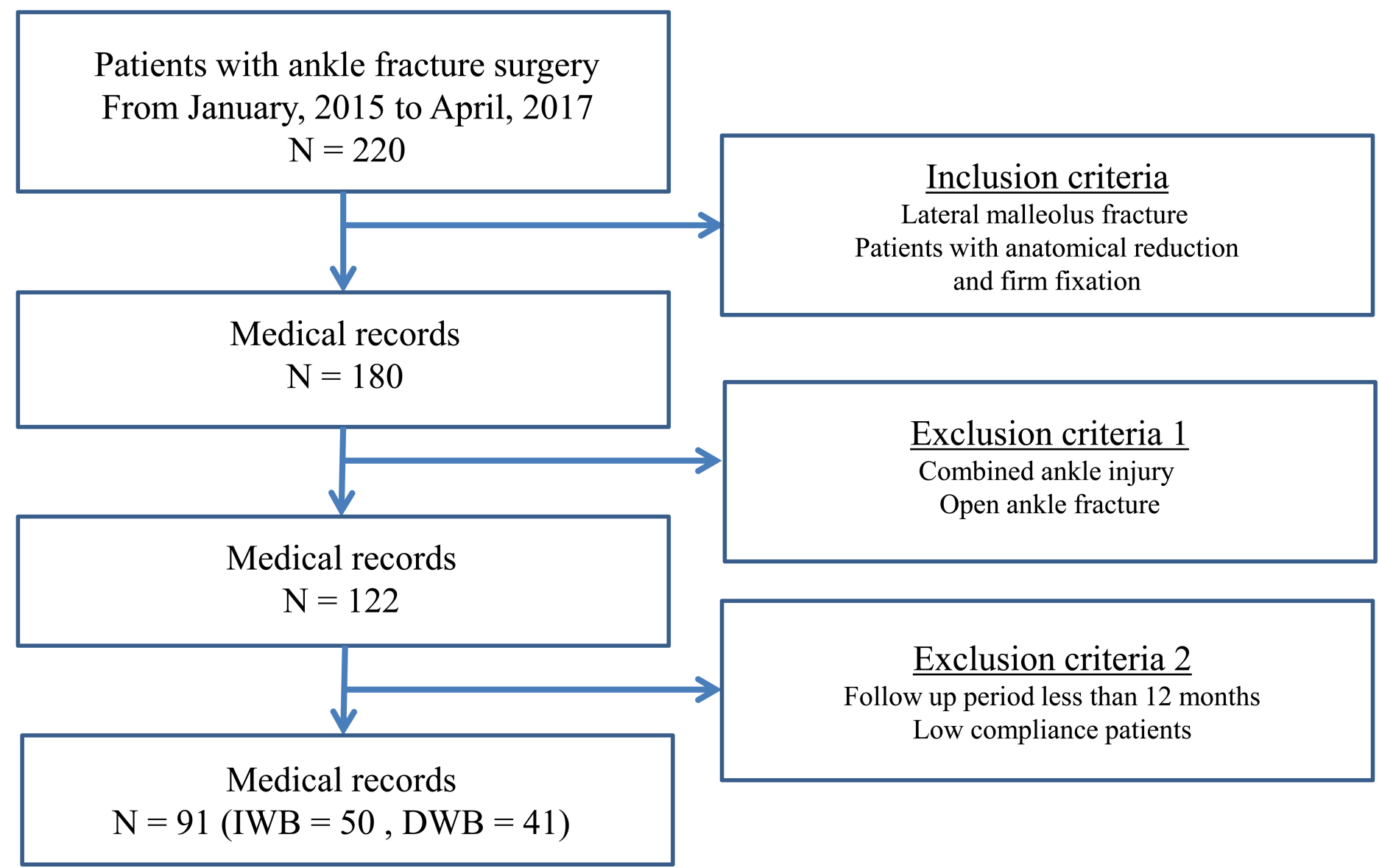

Figure 1

Inclusion and exclusion criteria

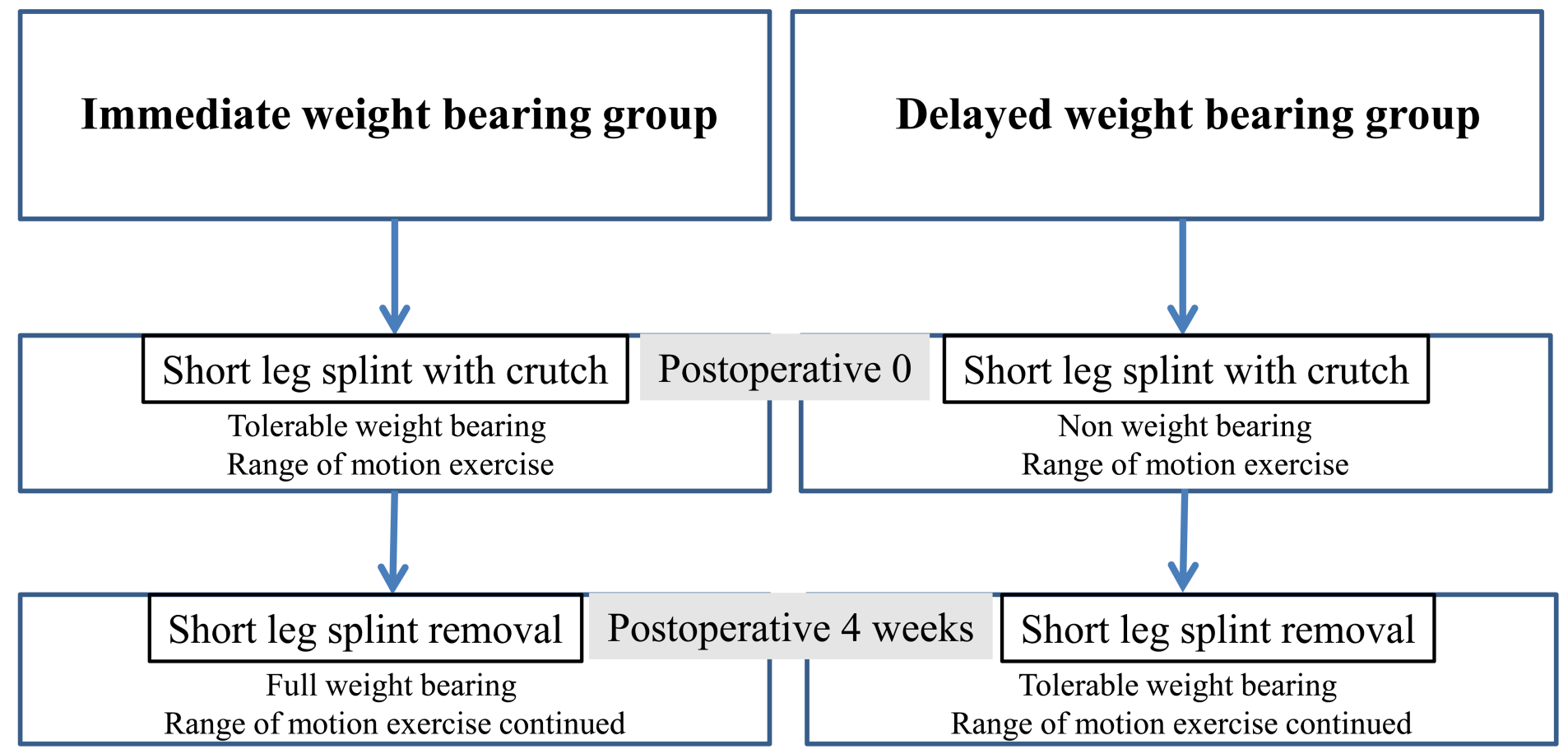


Figure 2

Rehabilitation protocol

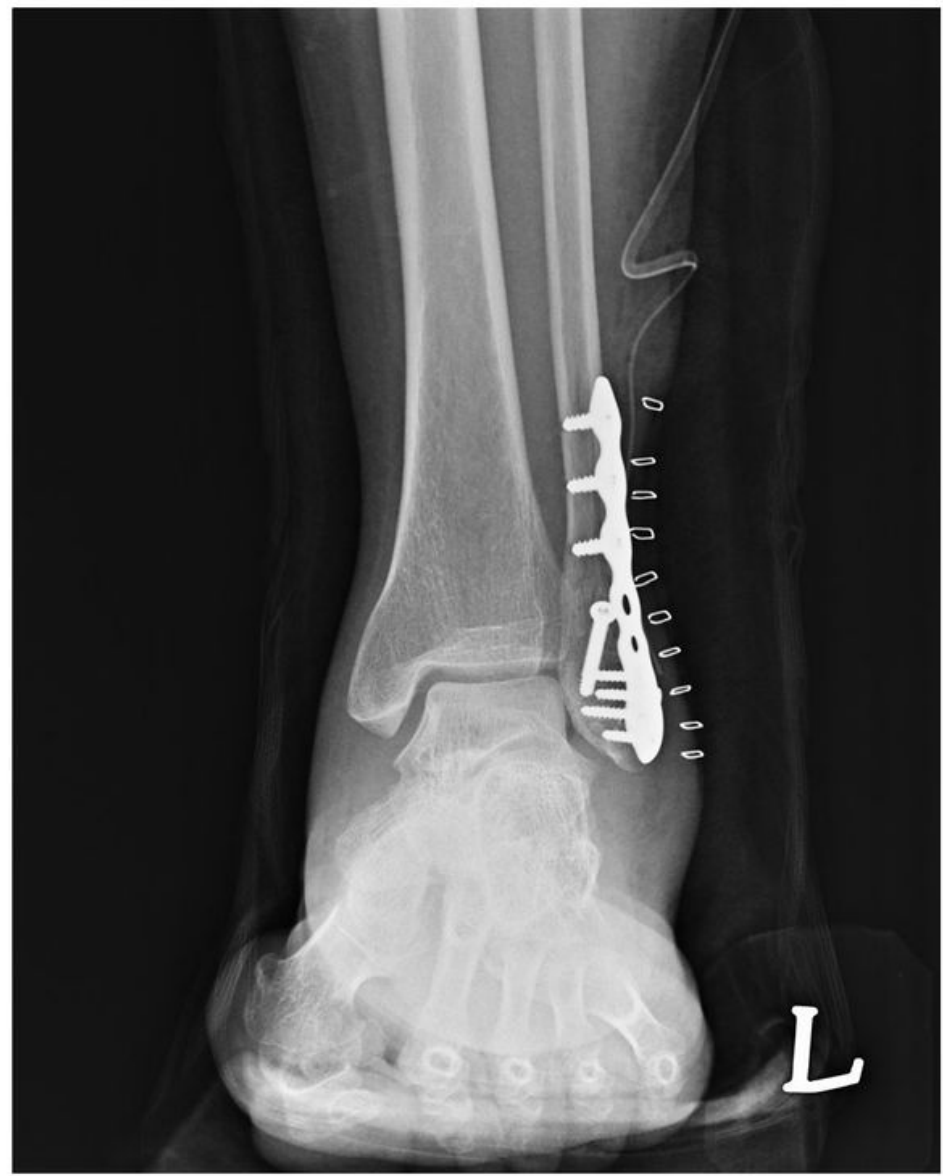

$\mathbf{A}$

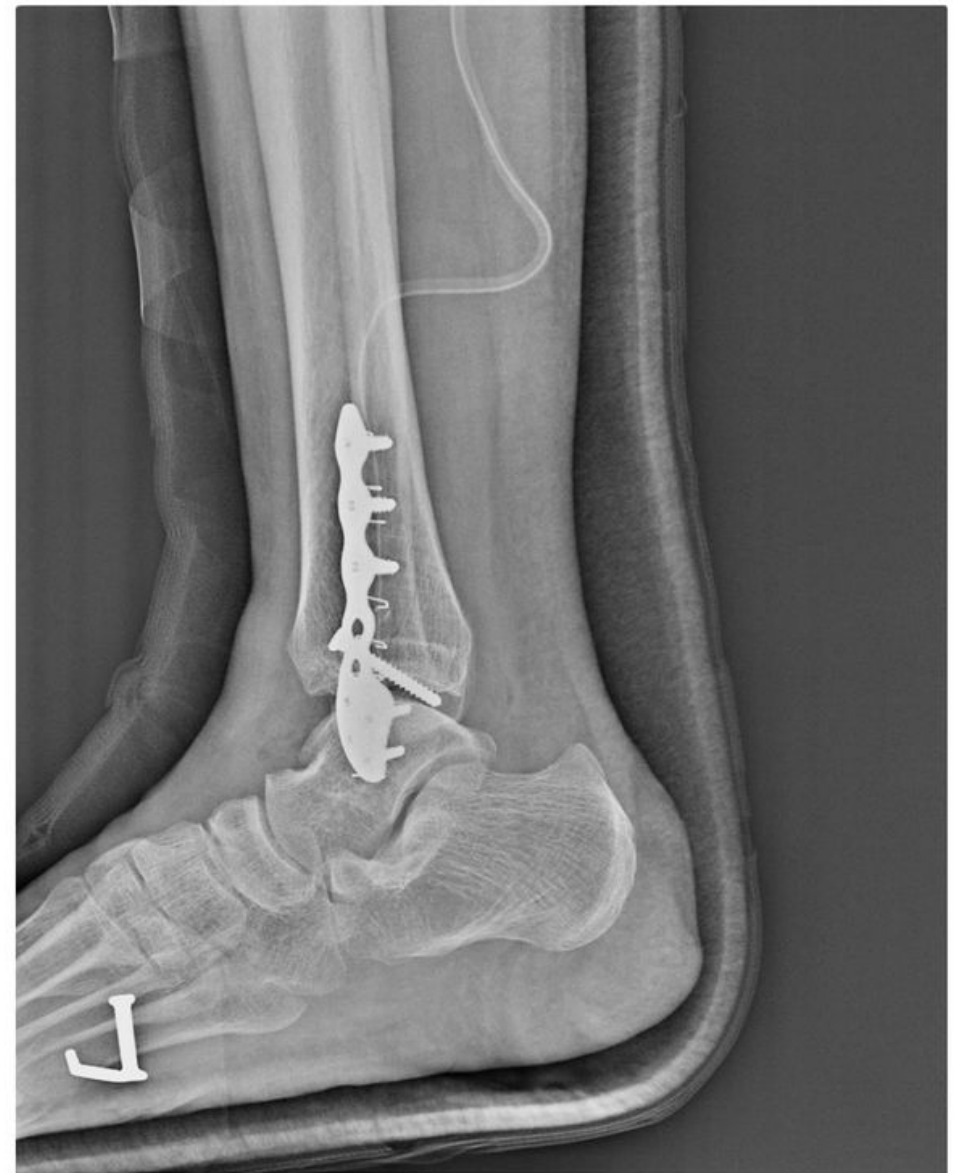

B

Figure 3

Postoperative ankle plain radiographs a. Anteroposterior view b. Lateral view 